\title{
Abundances of neutron-capture elements in G 24-25
}

\section{A halo-population $\mathrm{CH}$ subgiant ${ }^{\star, \star \star}$}

\author{
S. Liu ${ }^{1,2}$, P. E. Nissen ${ }^{3}$, W. J. Schuster ${ }^{4}$, G. Zhao ${ }^{1}$, Y. Q. Chen ${ }^{1}$, and Y. C. Liang ${ }^{1}$ \\ 1 Key Laboratory of Optical Astronomy, National Astronomical Observatories, CAS, 20A Datun Road, Chaoyang District, \\ 100012 Beijing, PR China \\ e-mail: gzhao@nao.cas.cn \\ 2 Graduate University of the Chinese Academy of Sciences, 19A Yuquan Road, Shijingshan District, 100049 Beijing, PR China \\ 3 Department of Physics and Astronomy, University of Aarhus, 8000 Aarhus C, Denmark \\ e-mail: pen@phys .au.dk \\ ${ }^{4}$ Observatorio Astronómico Nacional, Universidad Nacional Autónoma de México, Apartado Postal 877, CP 22800 Ensenada, \\ BC, Mexico \\ e-mail: schuster@astrosen.unam.mx
}

Received 18 August 2011 / Accepted 5 March 2012

\begin{abstract}
Aims. The differences between the neutron-capture element abundances of halo stars are important to our understanding of the nucleosynthesis of elements heavier than the iron group. We present a detailed abundance analysis of carbon and twelve neutron-capture elements from $\mathrm{Sr}$ up to $\mathrm{Pb}$ for a peculiar halo star $\mathrm{G} 24-25$ with $[\mathrm{Fe} / \mathrm{H}]=-1.4$ in order to probe its origin.

Methods. The equivalent widths of unblended lines are measured from high resolution NOT/FIES spectra and used to derive abundances based on Kurucz model atmospheres. In the case of $\mathrm{CH}, \mathrm{Pr}, \mathrm{Eu}, \mathrm{Gd}$, and $\mathrm{Pb}$ lines, the abundances are derived by fitting synthetic profiles to the observed spectra. Abundance analyses are performed both relative to the Sun and to a normal halo star G 16-20 that has similar stellar parameters as G 24-25.

Results. We find that G24-25 is a halo subgiant star with an unseen component. It has large overabundances of carbon and heavy $s$-process elements and mild overabundances of Eu and light $s$-process elements. This abundance distribution is consistent with that of a typical $\mathrm{CH}$ giant. The abundance pattern can be explained by mass transfer from a former asymptotic giant branch component, which is now a white dwarf.
\end{abstract}

Key words. stars: abundances - stars: atmospheres - stars: chemically peculiar - nuclear reactions, nucleosynthesis, abundances

\section{Introduction}

The heaviest elements in halo stars are mainly produced by neutron captures divided into the slow $(s-)$ and the rapid $(r-)$ processes, which have different reaction timescales. Among the $s$-process synthesized elements, lead $(\mathrm{Pb})$ is particularly interesting because it is the most abundant element in the third peak of the nuclei distribution in stars. However, $\mathrm{Pb}$ lines are very weak in low metallicity stars, and thus $\mathrm{Pb}$ abundances have not been measured for many stars compared with other $s$-process elements, such as $\mathrm{Ba}$. $\mathrm{Pb}$ is often detected for carbonenhanced stars with enhanced Ba abundances. For example, Bisterzo et al. (2006) compiled a catalog of $23 \mathrm{~Pb}$-rich stars found in the literature. Most of these stars have $[\mathrm{Fe} / \mathrm{H}]<-2$ and are carbon-enhanced metal-poor stars with $s$-process enhanced abundances (CEMP-s) or with $r$ - and $s$-process enhanced abundances $($ CEMP- $r / s)$. Eleven stars of the CEMP- $s /$ CEMP- $r / s$ compiled in Bisterzo's work are proven members of binary systems (McClure \& Woodsworth 1990; Aoki et al. 2001, 2002b; Lucatello et al. 2003; Cohen et al. 2003; Sivarani et al. 2004).

* Based on observations made with the Nordic Optical Telescope on La Palma.

$\star \star$ Table 2 is available in electronic form at http://www. aanda. org
In the past ten years, some studies also reported $\mathrm{Pb}$ measurements for $\mathrm{Ba}$ stars and $\mathrm{CH}$ giants and subgiants (Van Eck et al. 2001, 2003; Johnson \& Bolte 2004; Allen \& Barbuy 2006; Pereira \& Drake 2009, 2011; Goswami \& Aoki 2010). These classes of stars exhibit overabundances of $s$-process elements; in addition, the $\mathrm{CH}$ stars have high $\mathrm{C}$ abundances. Furthermore, these stars fall into different metallicity ranges and belong to different populations in the Galaxy. Most of the Ba stars are found in the disk with $[\mathrm{Fe} / \mathrm{H}]$ from -0.5 to +0.4 ; fewer than $6 \%$ of the Ba stars belong to the halo (Mennessier et al. 1997; Allen \& Barbuy 2006). The classical CH giants are clearly members of the halo population with $-1.5<[\mathrm{Fe} / \mathrm{H}]<-1.0$ (Vanture 1992a). The $\mathrm{CH}$ subgiants, i.e. stars with similar abundance distributions as the $\mathrm{CH}$ giants, but situated on either the main sequence or the subgiant branch, have typical $[\mathrm{Fe} / \mathrm{H}]$ values in the range from -0.5 to -0.2 and disk population kinematics (Luck \& Bond 1982, 1991; Sneden \& Parthasarathy 1983; Smith et al. 1993).

Systematic spectroscopic studies indicate that all $\mathrm{Ba}$ and $\mathrm{CH}$ stars belong to binary systems (McClure \& Woodsworth 1990). At low metallicity, $[\mathrm{Fe} / \mathrm{H}]<-1.8$, Lucatello et al. (2005) found that $68 \%$ of 19 CEMP- $s$ stars display evidence of radial velocity variations. This high frequency suggests that all CEMP- $s$ stars are metal-poor analogs of the classical $\mathrm{CH}$ giants. 
Table 1. Atmospheric parameters and radial velocities of G24-25 and $\mathrm{G} 16-20$.

\begin{tabular}{lccccc}
\hline \hline Star & $\begin{array}{c}T_{\text {eff }} \\
(\mathrm{K})\end{array}$ & $\log g$ & {$[\mathrm{Fe} / \mathrm{H}]$} & $\begin{array}{c}\xi \\
\left(\mathrm{km} \mathrm{s}^{-1}\right)\end{array}$ & $\begin{array}{c}v_{\text {rad }} \\
\left(\mathrm{km} \mathrm{s}^{-1}\right)\end{array}$ \\
\hline G 24-25 & 5828 & 3.86 & -1.40 & 1.20 & -312.9 \\
G 16-20 & 5625 & 3.64 & -1.42 & 1.50 & 170.8 \\
\hline
\end{tabular}

The origin of the enhanced carbon and $s$-process elements (including $\mathrm{Pb}$ ) in these stars could be explained by mass transfer from a former asymptotic giant branch (AGB) companion (now a white dwarf) in a binary system.

In a study of two distinct halo populations in the solar neighborhood, Nissen \& Schuster $(2010,2011)$ measured the abundance ratios and kinematics of 94 dwarf and subgiant stars and found a strong enhancement of $[\mathrm{Ba} / \mathrm{Fe}]$ for a halo star, $\mathrm{G} 24-25$, with $[\mathrm{Fe} / \mathrm{H}]=-1.4$. Further investigation of the spectrum identified a Pb line at $4057.8 \AA$, which is not seen for the rest of the stars. For example, the $\mathrm{Pb}$ feature at $4057.8 \AA$ cannot be detected in G 16-20, a halo star with a similar metallicity as G24-25. In addition, $\mathrm{G} 24-25$ has a strong $\mathrm{CH}$ band near $4300 \AA$, indicating that it is a $\mathrm{CH}$ subgiant. To our knowledge, this is the first known $\mathrm{CH}$ subgiant with a metallicity that is typical of the halo population. In this paper, we report our abundance measurements of $\mathrm{Pb}$ and other neutron-capture elements for this peculiar halo star. For comparison, G 16-20 is analyzed as a reference star. The abundance pattern is compared with other chemically peculiar stars in the literature, and simple AGB wind-accretion models are adopted to reproduce the measured abundances.

\section{Abundance analysis}

\subsection{Observational data and atmospheric parameters}

As described in Nissen \& Schuster (2010), the spectra of G 24-25 and G 16-20 were obtained at the $2.56 \mathrm{~m}$ Nordic Optical Telescope (NOT) using the FIbre fed Echelle Spectrograph (FIES) with a resolving power of $R \sim 40000$. The spectral range goes from $4000 \AA$ to $7000 \AA$, and the signal-to-noise ratio $(\mathrm{S} / \mathrm{N})$ equals about 170 at $5500 \AA$ but is only between 50 and 80 in the blue spectral region.

The stellar atmospheric parameters for G 24-25 and G 16-20 are taken from Nissen \& Schuster (2010) and given in Table 1, where the heliocentric radial velocities are also given. According to Nissen \& Schuster (2010), the total space velocities with respect to the local standard of rest are $315 \mathrm{~km} \mathrm{~s}^{-1}$ and $263 \mathrm{~km} \mathrm{~s}^{-1}$ for G 24-25 and G 16-20, respectively, which clearly classify both stars as members of the halo population.

\subsection{Line selection and atomic data}

The atomic lines were selected from previous abundance determinations of the heavy elements of stars, namely Sneden \& Parthasarathy (1983), Gratton \& Sneden (1994), Sneden et al. (1996), Reddy et al. (1997), Aoki et al. (2001, 2002b), Cowan et al. (2002), Sivarani et al. (2004), and Johnson \& Bolte (2004). The $\log g f$ values are primarily adopted from high-precision laboratory measurements; references are given in Table 2.

\subsection{Abundance calculations}

For most elements, the abundances are derived from equivalent widths (EWs) of unblended lines measured by fitting a

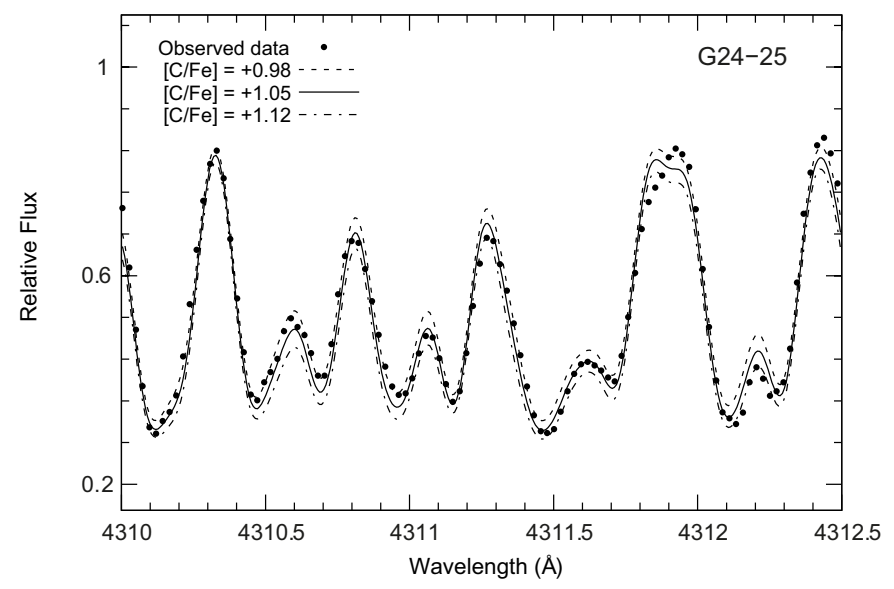

Fig. 1. The $\mathrm{CH}$ feature near $4310 \AA$. The observed spectrum of G $24-25$ is shown as bold dots. The solid line shows the synthetic spectrum corresponding to $[\mathrm{C} / \mathrm{Fe}]=1.05$. The dot-dashed and dashed lines, corresponding to $\Delta[\mathrm{C} / \mathrm{Fe}]= \pm 0.07$, are shown to illustrate the sensitivity of line strengths to variations in the carbon abundance.

Gaussian to the line profile. For blended lines and/or lines with significant hyperfine structure (HFS) and/or isotope splitting, the abundances are derived by spectrum synthesis. The model atmospheres of Kurucz (1993) are used, and local thermodynamic equilibrium (LTE) is assumed.

The carbon abundance is derived from the EWs of three unblended $\mathrm{C}_{\mathrm{I}}$ atomic lines providing $[\mathrm{C} / \mathrm{Fe}]=1.03$ and $[\mathrm{C} / \mathrm{Fe}]=-0.39$ for $\mathrm{G} 24-25$ and $\mathrm{G} \mathrm{16-20,}$, respectively. Compared to G 16-20, G 24-25 has clear carbon atomic lines and strong $\mathrm{CH}$ and $\mathrm{C}_{2}$ lines. From spectrum synthesis fitting of the CH A-X band of G 24-25 around $4310 \AA$ (Fig. 1), we obtained $[\mathrm{C} / \mathrm{Fe}]=1.05$ when using the $\mathrm{CH}$ molecular line data of Barklem et al. (2005). The abundances derived from the $\mathrm{C}_{\mathrm{I}}$ atomic lines and the $\mathrm{CH}$ A-X band are consistent, whereas Sivarani et al. (2004) reported that carbon abundances derived from $C_{\text {I }}$ lines are on average about $0.1-0.3$ dex higher than those obtained from the $\mathrm{CH}$ lines.

Since the CN $3800 \AA$ band is not covered in our spectra, we tried to derive the nitrogen abundance by spectrum synthesis fitting of the CN $4215 \AA$ band adopting molecular line data from Aoki et al. (2002a). The $\mathrm{CN}$ lines around $4215 \AA$ are quite weak $(E W<5 \mathrm{~m} \AA)$, and due to the rather low $\mathrm{S} / \mathrm{N}$ ratio in this spectral region, we can only get an upper limit $[\mathrm{N} / \mathrm{Fe}]<0.16 \mathrm{dex}$ for $\mathrm{G} 24-25$. In the case of $\mathrm{G} 16-20$ we get $[\mathrm{N} / \mathrm{Fe}]<0.0$ dex.

The abundances of the light $s$-process elements $\mathrm{Sr}^{1}$, $\mathrm{Y}$, and $\mathrm{Zr}$, as well as the heavy neutron-capture elements $\mathrm{Ba}, \mathrm{La}$, $\mathrm{Ce}, \mathrm{Nd}$, and $\mathrm{Sm}$ are derived from EW measurements, whereas spectrum synthesis is used for $\mathrm{Pr}, \mathrm{Eu}, \mathrm{Gd}$, and $\mathrm{Pb}$. The HFS of the Ba II lines at 5853.7, 6141.7, and 6496.9 $\AA$ is not taken into account since it is insignificant according to Sneden et al. (1996) and Mashonkina et al. (2010). Our Ba abundances are in good agreement with those in Nissen \& Schuster (2011), who also found that the effects of HFS on the Ba lines are negligible. The La II and Sm II lines are quite weak, and we do not include any HFS.

The collisional broadening of lines induced by neutral hydrogen is also considered. The width cross-sections of the $\mathrm{C}_{\mathrm{I}}$,

\footnotetext{
1 We do not include the Sr II line at $4161.8 \AA$, because the abundance inferred from that line is 0.3 dex higher than the average for the other lines (Sneden et al. 1996; Aoki et al. 2001). We also avoid the $\mathrm{Sr}$ II $\lambda 4215.5$ line, which is blended with an $\mathrm{Fe}_{\mathrm{I}}$ line.
} 
S. Liu et al.: Abundances of neutron-capture elements in G 24-25
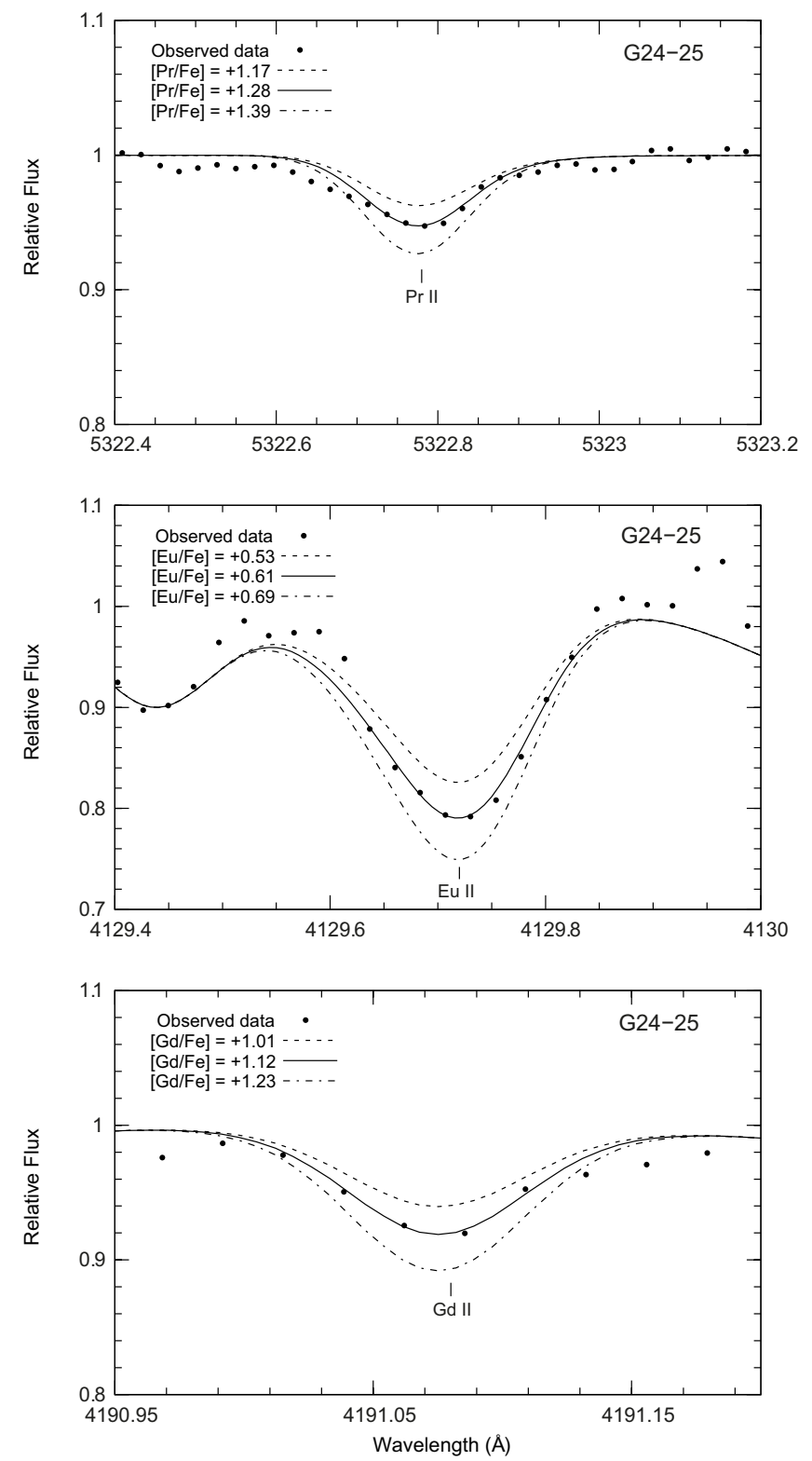

Fig. 2. Comparison of observed (bold dots) and synthetic profiles of the spectral lines of the heavy elements $\operatorname{Pr}$ (top panel), Eu (middle), and Gd (bottom). The respective elemental abundances corresponding to the full drawn lines are listed in Table 5. Two alternative synthetic spectra are shown to demonstrate the sensitivity of the line strength to the abundance.

Sr I, Sr II, and Ba II lines are taken from Anstee \& O’Mara (1995), Barklem \& O'Mara (1997, 2000), and Barklem et al. (2000). For the remaining lines, we follow Cohen et al. (2003) and adopt the Unsöld (1955) approximation to the van der Waals interaction constant enhanced by a factor of two. For the four strong lines, $\mathrm{Sr}_{\mathrm{II}} 4077.7 \AA$ and the Ba II lines at 5853.7, 6141.7, and $6496.9 \AA$, the effect on the derived abundances of the uncertainties in the damping constants is approximately 0.08 dex. However, most element abundances in our work are based on weak lines $(E W<60 \mathrm{~m} \AA)$, for which the influence on the results caused by the uncertainties in the collisional cross-sections is negligible. Hence, we do not discuss possible errors in the damping constants in Sect. 2.4.

The spectrum synthesis fits to the $\mathrm{Pr}, \mathrm{Eu}$, and $\mathrm{Gd}$ lines are shown in Fig. 2. The atomic and hyperfine structure data for

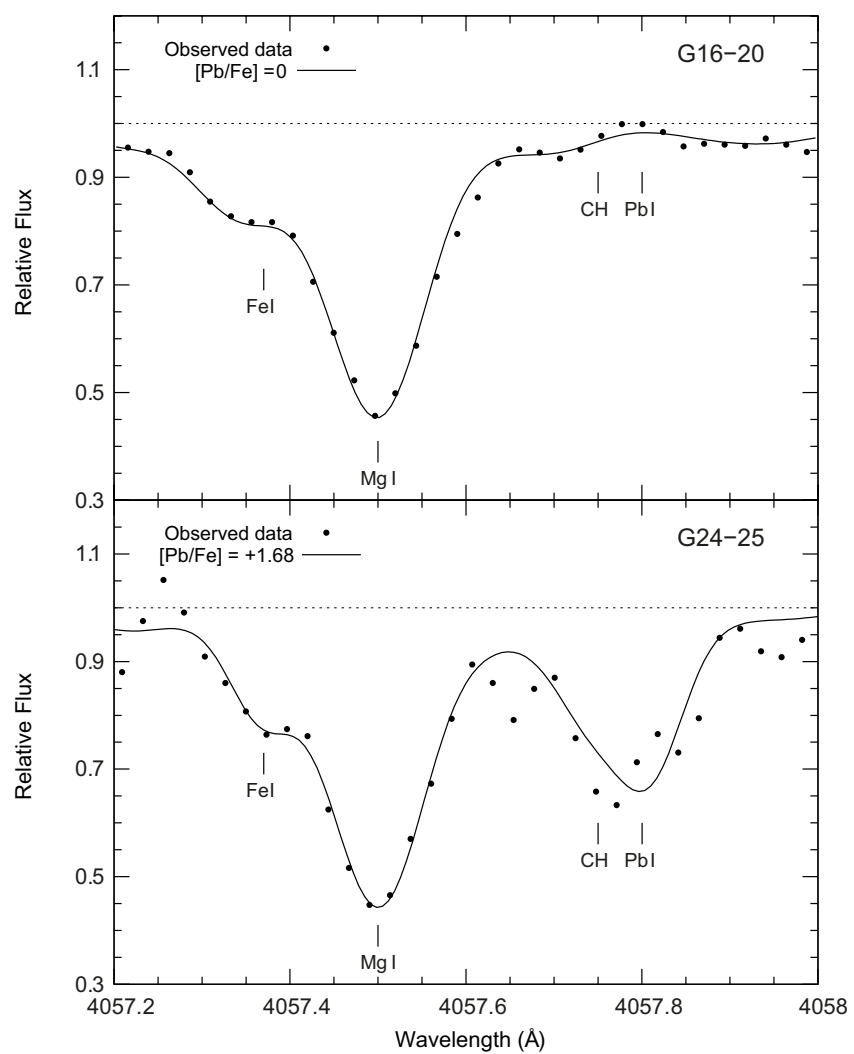

Fig. 3. Spectrum-synthesis fitting of the $\mathrm{Pb}$ I $\lambda 4057.81$ line for $\mathrm{G} 16-20$ and $\mathrm{G} 24-25$.

the Pri $5322.8 \AA$ and Eu II $4129.7 \AA$ lines are adopted from Mashonkina et al. (2009) and Mashonkina \& Gehren (2000), respectively. The $\log g f$ value for the Gd II line at $4191.1 \AA$ is taken from Den Hartog et al. (2006).

In the observed spectrum of G 24-25, we could detect one lead line, the $\mathrm{Pb}$ I $\lambda 4057.8$, which is too weak to be seen in the spectrum of G16-20 (Fig. 3). The $\log g f$ value is taken from Biémont et al. (2000) and the hyperfine data are taken from Aoki et al. (2001). Compared to G 16-20, a more normal neutroncapture star, it is clear that $\mathrm{G} 24-25$ exhibits a strong $\mathrm{Pb}$ line. The spectral region from $4057.6 \AA$ to $4058 \AA$ does not show a smooth line profile for G 24-25, but considering that the estimated $\mathrm{S} / \mathrm{N}$ around the $\mathrm{Pb}$ line is only about 60 , we propose that this is probably due to noise. Thus, a $\chi^{2}$ fitting of the ten observed data points from $4057.69 \AA$ to $4057.92 \AA$ was applied to determine the lead abundance. The $\chi^{2}$ was computed as described by Nissen et al. (1999), i.e.

$\chi^{2}=\frac{\sum\left(O_{i}-S_{i}\right)^{2}}{\sigma^{2}}$

where $O_{i}$ is the observed relative flux, $S_{i}$ the synthetic flux, and $\sigma=(S / N)^{-1}=1 / 60$. The value of $[\mathrm{Pb} / \mathrm{Fe}]$ was varied in steps of 0.02 dex to find the lowest $\chi^{2}$. The result is a parabolic variation in $\chi^{2}$ as shown in Fig. 4. The most probable value of $[\mathrm{Pb} / \mathrm{Fe}]$ corresponds to the minimum of $\chi^{2}$, and $\Delta \chi^{2}=1,4$, and 9 (the dashed horizontal lines) correspond to the 1-, 2-, and 3- $\sigma$ confidence limits of determining exclusively $[\mathrm{Pb} / \mathrm{Fe}]$. The spectrum synthesis fit to the $\mathrm{Pb}$ line for the abundance corresponding to the minimum $\chi^{2}([\mathrm{~Pb} / \mathrm{Fe}]=1.68)$ is shown as the solid line in Fig. 3. A Gaussian broadening function was applied to fit the observed spectrum in order to take the combined effect of instrumental, macro-turbulent, and rotational broadening into account. 


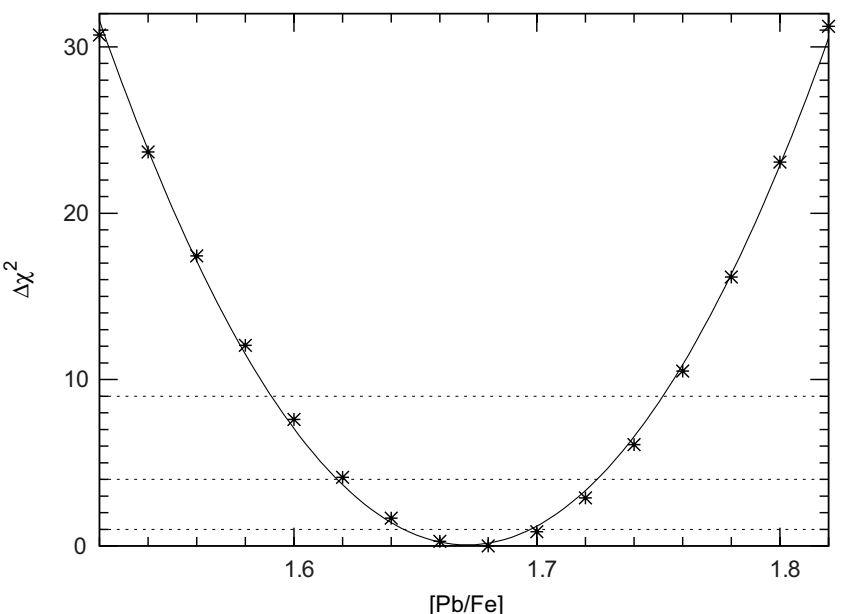

Fig. 4. The $\chi^{2}$ variation in the synthetic fit to ten observed data points from $4057.69 \AA$ to $4057.92 \AA$ applied to the determination of the lead abundance in G $24-25$.

The nearby spectral lines could be well-fitted in this way leading to a FWHM of the Gaussian function of $7.8 \pm 1 \mathrm{~km} \mathrm{~s}^{-1}$. The corresponding uncertainty in $[\mathrm{Pb} / \mathrm{Fe}]$ is around $0.04 \mathrm{dex}$.

\subsection{Error analysis}

The uncertainties in the abundances arise from random and systematic errors. The random errors associated with the errors in the $\log g f$ values and the EW measurements can be estimated from the line-to-line scatter in the abundances of a given element, based on many lines. The random error in the mean abundance is $\sigma_{\mathrm{EW}} / \sqrt{N}$, where $N$ is the number of lines studied. For the elements that have only one line, we use three times the uncertainty given by Cayrel (1988) by taking into consideration the error in the continuum rectification. For instance, Sr I $4607.34 \AA$ with $S / N=130$ leads to an error of $0.6 \mathrm{~m} \AA$ by using the formula (Eq. (7)) given by Cayrel (1988). We then estimate that the error in the EW measurement is $1.8 \mathrm{~m} \AA$ for $\operatorname{Sr}$ I $4607.34 \AA$, which results in an error of $0.06 \mathrm{dex}$ in the $\mathrm{Sr}$ I abundance calculation.

A significant contribution to the systematic errors comes from the uncertainties in the stellar atmospheric parameters adopted in the abundance determination. This was estimated by varying the model parameters with $\Delta T_{\text {eff }}=100 \mathrm{~K}, \Delta \log g=$ $0.10 \mathrm{dex}, \Delta[\mathrm{Fe} / \mathrm{H}]=0.1 \mathrm{dex}$, and $\Delta \xi=0.30 \mathrm{~km} \mathrm{~s}^{-1}$. The corresponding abundance uncertainties are listed in Tables 3 and 4, and the total uncertainty for each element is estimated by the quadratic sum of the atmospheric and random errors.

An additional systematic error in the derived abundances relative to the solar abundances is introduced by our LTE assumption and the use of one-dimensional model atmospheres. These errors tend, however, to cancel for the abundances of G 24-25 relative to G 16-20, because the two stars have similar atmospheric parameters and also comparable abundances of $\mathrm{Fe}$ and the $\alpha$-capture elements.

\section{Results and discussion}

\subsection{Abundance results}

The abundances for 13 elements are presented in Table 5, along with the number of lines on which they are based, the adopted solar abundances, $\log \varepsilon=\log \left(N_{\mathrm{X}} / N_{\mathrm{H}}\right)+12$, and both [X/Fe]
Table 3. Abundance uncertainties for neutron-capture elements in G 24-25.

\begin{tabular}{lrrrrrr}
\hline \hline Elem. & \multicolumn{1}{c}{$\Delta T$} & $\Delta \log g$ & \multicolumn{1}{c}{$\Delta[\mathrm{Fe} / \mathrm{H}]$} & \multicolumn{1}{c}{$\Delta \xi$} & $\frac{\sigma_{\mathrm{EW}}}{\sqrt{N}}$ & $\sigma_{\text {tot }}$ \\
& $+100 \mathrm{~K}$ & +0.10 & \multicolumn{1}{c}{+0.10} & +0.30 & & \\
\hline $\mathrm{C}_{\text {I }}$ & -0.06 & 0.03 & -0.01 & -0.01 & 0.03 & 0.07 \\
$\mathrm{Sr}_{\text {I }}$ & 0.08 & 0.00 & 0.01 & -0.02 & 0.06 & 0.10 \\
$\mathrm{Sr}_{\text {II }}$ & 0.06 & 0.01 & 0.03 & -0.02 & 0.04 & 0.08 \\
$\mathrm{Y}_{\text {II }}$ & 0.05 & 0.03 & 0.00 & -0.06 & 0.02 & 0.09 \\
$\mathrm{Zr}_{\text {I }}$ & 0.08 & 0.00 & 0.00 & -0.01 & 0.07 & 0.11 \\
$\mathrm{Zr}_{\text {II }}$ & 0.04 & 0.04 & 0.00 & -0.04 & 0.02 & 0.07 \\
$\mathrm{Ba}_{\text {II }}$ & 0.09 & -0.02 & 0.01 & -0.10 & 0.02 & 0.14 \\
$\mathrm{La}_{\text {II }}$ & 0.05 & 0.02 & 0.00 & -0.06 & 0.03 & 0.08 \\
$\mathrm{C}_{\text {II }}$ & 0.05 & 0.03 & 0.00 & -0.05 & 0.01 & 0.08 \\
$\mathrm{Pr}_{\text {II }}$ & 0.04 & 0.04 & -0.02 & -0.03 & 0.09 & 0.11 \\
$\mathrm{Nd}_{\text {II }}$ & 0.06 & 0.03 & 0.01 & -0.03 & 0.01 & 0.08 \\
$\mathrm{Sm}_{\text {II }}$ & 0.06 & 0.03 & 0.01 & -0.02 & 0.03 & 0.08 \\
$\mathrm{Eu}_{\text {II }}$ & 0.06 & 0.04 & -0.01 & 0.03 & 0.03 & 0.08 \\
$\mathrm{Gd}_{\text {II }}$ & 0.05 & 0.03 & -0.01 & -0.04 & 0.09 & 0.11 \\
$\mathrm{~Pb}_{\text {I }}$ & 0.09 & 0.03 & -0.01 & 0.04 & 0.08 & 0.13 \\
\hline
\end{tabular}

Table 4. Abundance uncertainties for neutron-capture elements in G 16-20.

\begin{tabular}{lcccrcc}
\hline \hline Elem. & \multicolumn{1}{c}{$\Delta T$} & $\Delta \log g$ & \multicolumn{1}{c}{$\Delta[\mathrm{Fe} / \mathrm{H}]$} & \multicolumn{1}{c}{$\Delta \xi$} & $\frac{\sigma_{\mathrm{EW}}}{\sqrt{N}}$ & $\sigma_{\text {tot }}$ \\
& $+100 \mathrm{~K}$ & +0.10 & +0.10 & +0.30 & & \\
\hline $\mathrm{C}_{\text {I }}$ & -0.06 & 0.04 & -0.01 & 0.00 & 0.09 & 0.12 \\
$\mathrm{Sr}_{\text {I }}$ & 0.09 & 0.00 & 0.00 & -0.01 & 0.07 & 0.11 \\
$\mathrm{Sr}_{\text {II }}$ & 0.05 & 0.01 & 0.01 & -0.04 & 0.03 & 0.07 \\
$\mathrm{Y}_{\text {II }}$ & 0.04 & 0.04 & 0.01 & -0.01 & 0.02 & 0.06 \\
$\mathrm{Zr}_{\text {II }}$ & 0.04 & 0.03 & 0.01 & -0.02 & 0.03 & 0.06 \\
$\mathrm{Ba}_{\text {II }}$ & 0.06 & 0.06 & 0.01 & -0.08 & 0.03 & 0.12 \\
$\mathrm{La}_{\text {II }}$ & 0.05 & 0.03 & 0.01 & -0.01 & 0.02 & 0.06 \\
$\mathrm{Ce}_{\text {II }}$ & 0.06 & 0.04 & 0.01 & 0.00 & 0.02 & 0.08 \\
$\mathrm{Nd}_{\text {II }}$ & 0.06 & 0.04 & 0.01 & 0.00 & 0.04 & 0.08 \\
$\mathrm{Sm}_{\text {II }}$ & 0.06 & 0.04 & 0.01 & -0.01 & 0.02 & 0.08 \\
$\mathrm{Eu}_{\text {II }}$ & 0.05 & 0.03 & -0.01 & 0.02 & 0.03 & 0.07 \\
$\mathrm{Gd}_{\text {II }}$ & 0.06 & 0.04 & -0.01 & -0.03 & 0.09 & 0.12 \\
\hline & & & & & & \\
\hline
\end{tabular}

and $[\mathrm{X} / \mathrm{Ba}]$ for each species. We adopted the standard solar abundances of Asplund et al. (2009) as a reference for the various elements. As seen, all of the 13 elements studied are enhanced in G 24-25 relative to both the Sun and the reference star G 16-20.

In Fig. 5, we present the abundance patterns of G 24-25 and G 16-20. The abundances of $\mathrm{Na}, \mathrm{Mg}, \mathrm{Si}, \mathrm{Ca}, \mathrm{Ti}, \mathrm{Cr}, \mathrm{Ni}, \mathrm{Mn}, \mathrm{Cu}$, and $\mathrm{Zn}$ are adopted from Nissen \& Schuster $(2010,2011)$. The two stars have similar behaviors for both the $\alpha$ - and iron-peak elements but show obvious differences for $\mathrm{C}$ and heavy neutroncapture elements. This can be attributed to the chemical peculiarity of G 24-25. In particular, the second-peak s-process elements (Ba, La, Ce, Pr, Nd, Sm, Gd) are more enhanced than the first-peak $s$-process elements ( $\mathrm{Sr}, \mathrm{Y}, \mathrm{Zr}$ ) in G 24-25, which also has a strong carbon enhancement.

Taking into account the most recent research on metalpoor stars, Beers \& Christlieb (2005) divided metal-poor stars into various subclasses. According to their definitions, G 24-25, which is both carbon- and barium- enhanced with $[\mathrm{C} / \mathrm{Fe}]>+1.0$ and $[\mathrm{Ba} / \mathrm{Fe}]>+1.0$ along with $[\mathrm{Ba} / \mathrm{Eu}]>+0.5$, can be classified as CEMP-s. However, most CEMP- $s$ stars found in the literature have $[\mathrm{Fe} / \mathrm{H}]<-2$, which is more metal-deficient than $\mathrm{G} 24-25$ with $[\mathrm{Fe} / \mathrm{H}]=-1.4$. On the other hand, $\mathrm{CH}$ stars have higher metallicities than CEMP stars and occur in binary systems. Given that G 24-25 is a single-lined spectroscopic binary (Latham et al. 2002), we suggest that G 24-25 should be classified as a $\mathrm{CH}$ subgiant, although it has a lower metallicity than 
Table 5. Abundances of neutron-capture elements of G 24-25 and G 16-20.

\begin{tabular}{lcccccccccc}
\hline \hline Elem. & $Z$ & $\log \varepsilon_{\odot}$ & \multicolumn{4}{c}{$\mathrm{G} 24-25$} \\
& & & $N_{\text {lines }}$ & $\log \varepsilon$ & {$[\mathrm{X} / \mathrm{Fe}]$} & {$[\mathrm{X} / \mathrm{Ba}]$} & $N_{\text {lines }}$ & $\log \varepsilon$ & {$[\mathrm{X} / \mathrm{Fe}]$} & {$[\mathrm{X} / \mathrm{Ba}]$} \\
\hline $\mathrm{C}_{\text {I }}$ & 6 & 8.39 & 3 & 8.03 & 1.03 & -0.32 & 1 & $6.58:$ & $-0.39:$ & $-0.21:$ \\
$\mathrm{Sr}_{\mathrm{I}}$ & 38 & 2.92 & 1 & 1.96 & 0.44 & -0.91 & 1 & 1.19 & -0.31 & -0.13 \\
$\mathrm{Sr}_{\text {II }}$ & 38 & 2.92 & 1 & 1.79 & 0.27 & -1.08 & 1 & 1.09 & -0.41 & -0.23 \\
$\mathrm{Y}_{\text {II }}$ & 39 & 2.21 & 8 & 1.51 & 0.70 & -0.65 & 7 & 0.59 & -0.20 & -0.02 \\
$\mathrm{Zr}_{\text {I }}$ & 40 & 2.59 & 1 & 2.14 & 0.95 & -0.40 & $\ldots$ & $\ldots$ & $\ldots$ & $\ldots$ \\
$\mathrm{Zr}_{\text {II }}$ & 40 & 2.59 & 7 & 2.14 & 0.95 & -0.40 & 3 & 1.40 & 0.23 & 0.38 \\
$\mathrm{Ba}_{\text {II }}$ & 56 & 2.17 & 3 & 2.12 & 1.35 & $\ldots$ & 3 & 0.57 & -0.18 & $\ldots$ \\
$\mathrm{La}_{\text {II }}$ & 57 & 1.13 & 5 & 1.28 & 1.55 & 0.20 & 2 & 0.06 & 0.35 & 0.53 \\
$\mathrm{Ce}_{\text {II }}$ & 58 & 1.58 & 22 & 1.77 & 1.59 & 0.24 & 6 & 0.31 & 0.15 & 0.33 \\
$\mathrm{Pr}_{\text {II }}$ & 59 & 0.71 & 1 & 0.59 & 1.28 & -0.07 & 1 & $<-0.45$ & $<0.26$ & $<0.44$ \\
$\mathrm{Nd}_{\text {II }}$ & 60 & 1.45 & 14 & 1.52 & 1.47 & 0.12 & 6 & 0.33 & 0.30 & 0.48 \\
$\mathrm{Sm}_{\text {II }}$ & 62 & 1.01 & 9 & 1.01 & 1.40 & 0.05 & 5 & -0.07 & 0.34 & 0.52 \\
$\mathrm{Eu}_{\text {II }}$ & 63 & 0.52 & 2 & -0.27 & 0.61 & -0.74 & 2 & -0.53 & 0.37 & 0.55 \\
$\mathrm{Gd}_{\text {II }}$ & 64 & 1.12 & 1 & 0.84 & 1.12 & -0.23 & 1 & 0.08 & 0.38 & 0.56 \\
$\mathrm{~Pb}_{\text {I }}$ & 82 & 2.00 & 1 & 2.28 & 1.68 & 0.33 & 1 & $<0.60$ & $<0.00$ & $<0.18$ \\
\hline
\end{tabular}

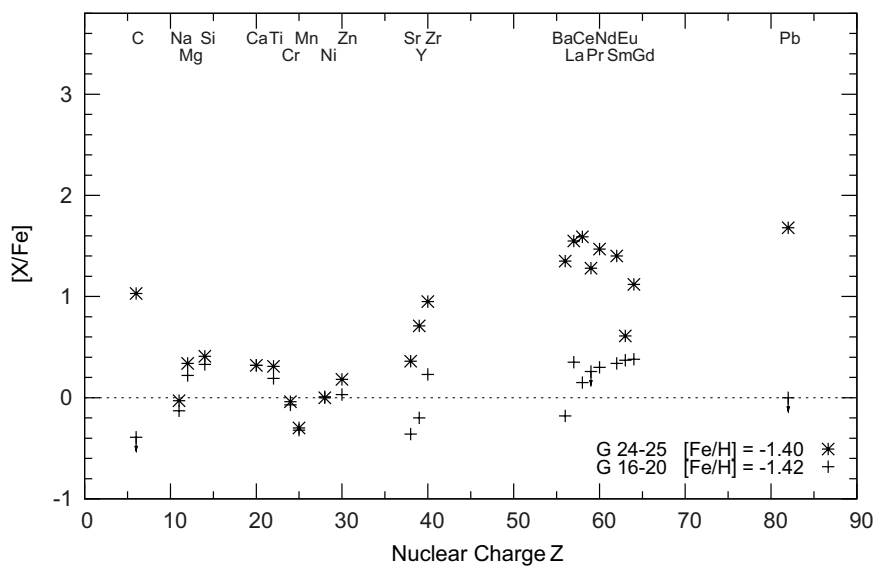

Fig. 5. Comparison of abundances in G 24-25 and G 16-20.

the classical $\mathrm{CH}$ subgiants belonging to the Galactic disk. In this context, the $\mathrm{C}$ and $s$-process element enhancements are consistent with mass transfer from a former AGB binary companion (McClure \& Woodsworth 1990).

\subsection{Comparing the abundance pattern to model predictions}

To investigate whether the abundance pattern of G 24-25 can be explained by mass transfer from a former AGB companion in a binary system, the observed abundances are compared with the values predicted by theoretical models. In this comparison, the ratios of the abundances of G 24-25 to those of the reference star G 16-20 are used to ensure that the effects of Galactic chemical evolution are minimized.

Low-mass AGB stars $\left(1.3 \lesssim M / M_{\odot} \lesssim 3\right)$ are affected by recurrent thermal pulses in the He shell leading to correlated enhancements of freshly synthesized ${ }^{12} \mathrm{C}$ and $s$-process elements in their photospheres (Straniero et al. 1995; Gallino et al. 1998). In addition, the more massive AGB stars undergo hot bottom burning where ${ }^{12} \mathrm{C}$ is converted into ${ }^{14} \mathrm{~N}$. The minimum mass for this process is somewhat uncertain, but at the metallicity of G 24-25, corresponding to $Z \simeq 0.001$, Karakas \& Lattanzio (2007) estimate it to be around $3.5 M_{\odot}$ (see their Table 1). Below this mass limit, an AGB star can have a C-rich atmosphere with no enhancement of nitrogen as found in the case of G 24-25 $([\mathrm{N} / \mathrm{Fe}]<0.16)$. Hence, the mass of the AGB star that is responsible for the abundance anomalies of G 24-25 is unlikely to be higher than about $3 M_{\odot}$; a simple consideration of the standard initial mass function implies that this mass is more likely to be around $1.5 M_{\odot}$.

Bisterzo et al. (2010) present theoretical predictions of an updated low-mass AGB stellar nucleosynthesis model at different metallicities. For a model with a given initial AGB mass and metallicity, they provide the abundances of all elements from carbon to bismuth in the envelope. They apply the analysis to CEMP- $s$ stars in Bisterzo et al. (2011) and get a good fit to the observed abundances by varying the ${ }^{13} \mathrm{C}$-pocket profile and dilution factor. Unfortunately, the calculated model data published in Bisterzo et al. $(2010,2011)$ are limited (only two ${ }^{13} \mathrm{C}$-pocket cases are available for the mass and metallicity we need, $M_{\mathrm{ini}}^{\mathrm{AGB}}=$ $1.5 M_{\odot}$ and $\left.[\mathrm{Fe} / \mathrm{H}]=-1.3\right)$, and the model prediction does not closely match the abundances of G 24-25.

Cristallo et al. (2009) also present a homogeneous set of calculations of low-mass AGB models at different metallicities, which they extend to create a database of AGB nucleosynthesis predictions and yields, called the FRANEC Repository of Updated Isotopic Tables \& Yield (FRUITY) database (Cristallo et al. 2011). This database is an online interactive interface of predictions for the surface composition of AGB stars undergoing the third dredge-up (TDU) when choosing different combinations of initial mass and metallicity. The first set of 28 AGB models available from FRUITY covers the masses $1.5 \leq M / M_{\odot} \leq 3.0$ and metallicities $1 \times 10^{-3} \leq Z \leq 2 \times 10^{-2}$. The stellar models of the FRUITY database were computed based on the updated FRANEC code (Chieffi et al. 1998). Carbon-enhanced opacity tables were adopted to take into account the effects of TDU episodes. The computation ends when the minimum envelope mass for TDU occurs. For our purpose, we adopted the AGB model computation from FRUITY for an initial mass $M=1.5 M_{\odot}$ and $Z=0.001$ (corresponding to $[\mathrm{Fe} / \mathrm{H}]=-1.3$ ) and scaled the whole abundance pattern down to our observational data by a factor of 1.6. These AGB model predictions for neutron-capture elements from $\mathrm{Sr}$ to $\mathrm{Pb}$ is presented by a solid line in Fig. 6. For most elements, the observed abundances are fitted quite well, but the Eu abundance is significantly overabundant according to the model prediction.

Alternatively, the parametric AGB model for metal-poor stars from Zhang et al. (2006) and Cui et al. (2010) has been used to predict the abundance pattern of G 24-25, although this model is only available for a mass of $3 M_{\odot}$ and $Z=0.0001$. 


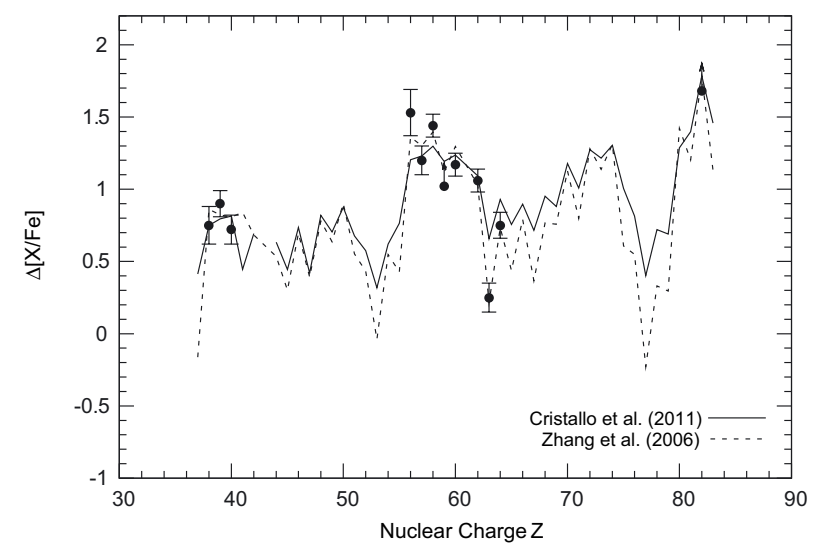

Fig. 6. The observed abundances of the neutron-capture elements of G 24-25 relative to G16-20 in comparison with two AGB model predictions.

They adopted the model for metal-poor stars presented by Aoki et al. (2001) after updating many of the neutron-capture rates according to Bao et al. (2000). This approach is not based on detailed stellar evolution models, but has been used to successfully explain the abundance patterns of some very metal-poor stars. There are three parameters in the model: the neutron exposure per thermal pulse, $\Delta \tau$, the overlap factor, $r$, and the component coefficient of the $s$-process, $C_{s}$. In the case of multiple subsequent exposures, the mean neutron exposure is given by $\tau_{0}=-\Delta \tau / \ln r$.

In their model, the convective He shell and the envelope of the giant at some time on the AGB, will be overabundant in heavy elements by factors of $f_{\text {shell }}$ and $f_{\text {env, } 1}$, respectively, with respect to Solar System abundances normalized to the values of the metallicity, $Z$. The approximate relation between $f_{\text {shell }}$ and $f_{\text {env, }, 1}$ is given by Eq. (3) of Zhang et al. (2006), where $\Delta M_{\mathrm{dr}}$ is the total mass dredged up from the He shell into the envelope of the AGB star, and $M_{1}^{\mathrm{e}}$ is the envelope mass. Given a mass of $3 M_{\odot}$ and $Z=0.0001$ for the AGB star, $\Delta M_{\mathrm{dr}} / M_{1}^{\mathrm{e}}$ is $1 / 9$ according to Karakas \& Lattanzio (2007).

For a given $s$-process element, the overabundance factor $f_{\text {env, } 2}$ in the companion star envelope can be approximately related to the overabundance factor $f_{\text {env, } 1}$ by Eq. (4) of Zhang et al. (2006), where $\Delta M_{2}$ is the amount of matter accreted by the companion star from the AGB progenitor, and $M_{2}^{\mathrm{e}}$ is the envelope mass of the accreting star. The component coefficient, $C_{s}=f_{\text {env }, 2} / f_{\text {shell }}$, is then computed from Eq. (5) of Zhang et al. (2006).

The closest possible match between the observed and predicted abundances is obtained for the following values of the three parameters: overlap factor, $r=0.50$, neutron exposure, $\Delta \tau=0.26 \mathrm{mb}^{-1}$ (i.e. a mean neutron exposure $\tau_{0}=0.375 \mathrm{mb}^{-1}$ ), and $s$-process component coefficient, $C_{s}=0.0012384$. The predicted abundance pattern is given as the dashed line in Fig. 6.

To highlight the AGB contribution, Fig. 6 shows $[\mathrm{X} / \mathrm{Fe}]_{\mathrm{G} 24-25}$ minus $[\mathrm{X} / \mathrm{Fe}]_{\mathrm{G} 16-20}$ as a measure of the abundances of the atmospheric material that has been accreted by G 24-25 from its donor star. We note, however, that the values plotted for $[\mathrm{Pr} / \mathrm{Fe}]$ and $[\mathrm{Pb} / \mathrm{Fe}]$ are lower limits to the difference between G 24-25 and G 16-20, because the $\mathrm{Pr}$ and $\mathrm{Pb}$ lines could not be detected in G 16-20, hence we have only upper limits to both $[\mathrm{Pr} / \mathrm{Fe}]$ and $[\mathrm{Pb} / \mathrm{Fe}]$ for this reference star. From Fig. 6, we can see that the observed abundance distribution is consistent with the two model predictions, except that a too high Eu abundance is predicted by the FRUITY model. The

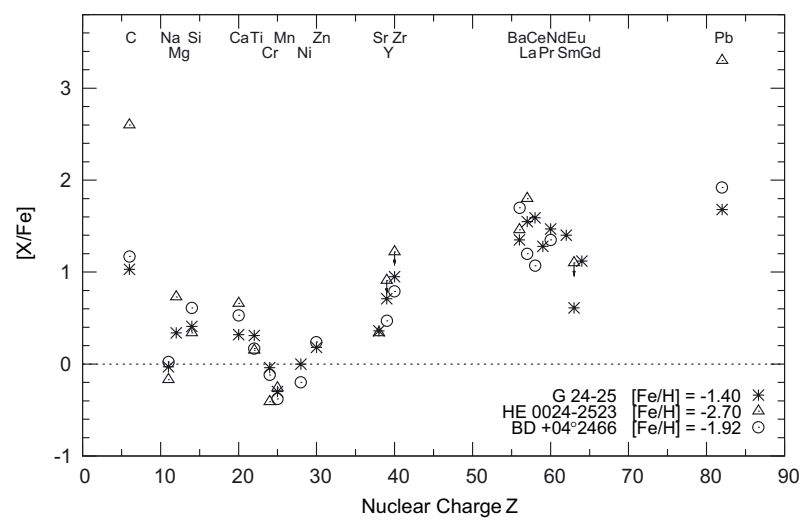

Fig. 7. Comparison of G 24-25 with HE 0024-2523 (CEMP- $s$ dwarf) and $\mathrm{BD}+04^{\circ} 2466$ ( $\mathrm{CH}$ giant).

consistency between the observed abundance distribution and the model predictions indicates that the overabundances of $s$-process elements in G 24-25 can be assumed to originate from mass transfer in a binary system containing material produced by an AGB companion.

\subsection{Comparison of G 24-25 with other peculiar stars}

It is also interesting to compare the abundance peculiarities of the neutron-capture elements in G24-25 with those of some well-studied CEMP-s or CH stars. We chose from the literature stars with as many heavy-element abundances as possible and atmospheric parameters close to those of G 24-25. Unfortunately, not one chemically peculiar star was found to satisfy all these criteria. Instead, two more metal-poor stars, HE 0024-2523 (Lucatello et al. 2003) and BD +04 2466 (Pereira \& Drake 2009), were considered, both of which have 17 elements available for comparison and are members of binary systems. HE 0024-2523 is a CEMP-s dwarf star with $[\mathrm{Fe} / \mathrm{H}]=-2.7$, and $\mathrm{BD}+04^{\circ} 2466$ is a classical $\mathrm{CH}$ giant with $[\mathrm{Fe} / \mathrm{H}]=-1.9$. As seen from Fig. 7, G24-25 shows much the same abundance pattern as the two stars, although G 24-25 is closer to $\mathrm{BD}+04^{\circ} 2466$, the classic $\mathrm{CH}$ star, than to HE0024-2523. We note that HE0024-2523 was classified as a lead star with an extreme carbon enhancement given by $[\mathrm{Pb} / \mathrm{Fe}]=+3.3$ and $[\mathrm{Pb} / \mathrm{Ba}]=+1.9$.

\subsection{Comparison of $[\mathrm{s} / \mathrm{Fe}]$ versus $[\mathrm{Fe} / \mathrm{H}]$ for chemically peculiar binary stars}

Due to the similar formation mechanisms, we have compared G 24-25 with other classes of chemically peculiar binary stars, i.e. the CEMP dwarfs, subgiants and giants; the $\mathrm{CH}$ subgiants and the classical $\mathrm{CH}$ giants; the Ba dwarfs and giants. The selected binary stars either have derived orbit solutions or exhibit radial velocity variations beyond $3 \sigma$ (Lu et al. 1987; McClure \& Woodsworth 1990; Preston \& Sneden 2001; Aoki et al. 2003; Cohen et al. 2003; Lucatello et al. 2003; Pourbaix et al. 2004; Tsangarides et al. 2004). These various classes are indicated by different symbols in Fig. 8 , where $[h s / \mathrm{Fe}]$ and $[l s / \mathrm{Fe}]$ represent the average abundances relative to Fe of the second-peak elements (Ba, La, Ce, Nd, Sm) and the first-peak $s$-process elements $(\mathrm{Sr}, \mathrm{Y}, \mathrm{Zr})$, respectively. Pr and $\mathrm{Gd}$ are not included in the comparison because most works in the literature have no Pr or Gd measurements. We do not include the so called "metal-deficient Ba stars" (mdBa). In the past two decades, only 
S. Liu et al.: Abundances of neutron-capture elements in G 24-25

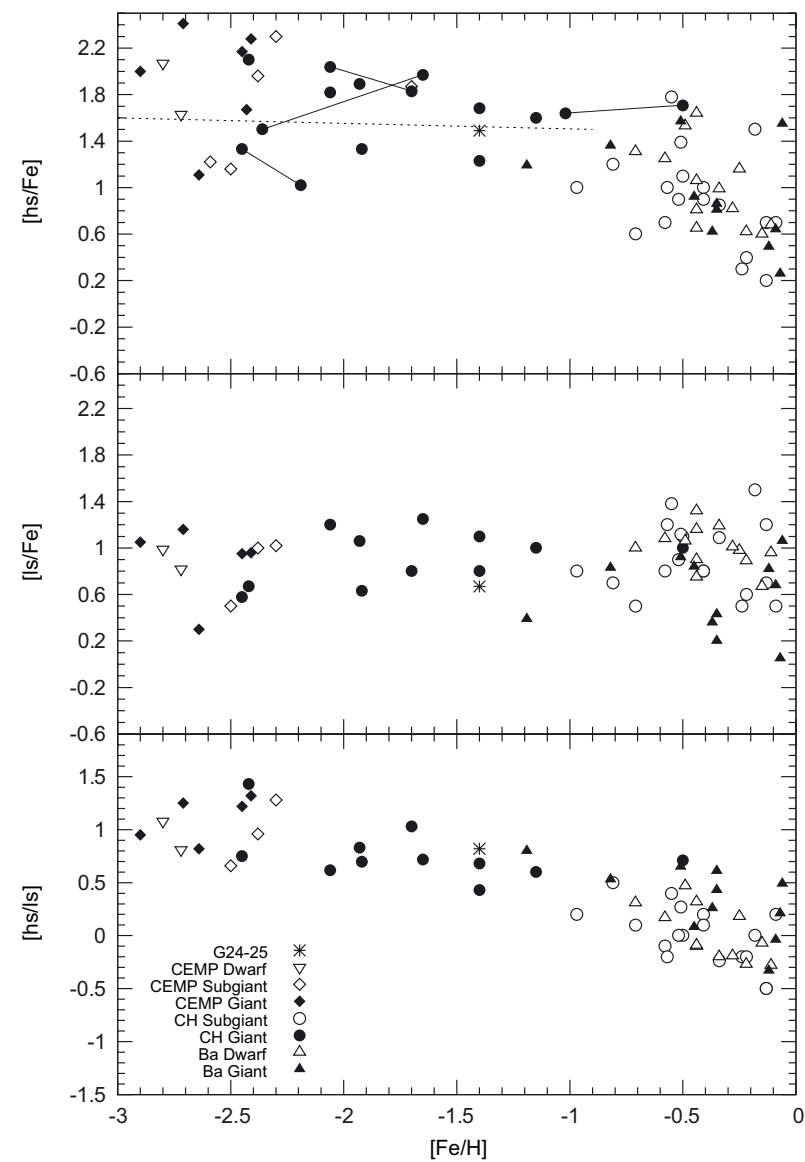

Fig. 8. $[h s / \mathrm{Fe}],[l s / \mathrm{Fe}]$ and $[h s / l s]$ vs. $[\mathrm{Fe} / \mathrm{H}]$ are shown in the three panels, from top to bottom, respectively. Dashed line separates stars belonging to the high and the low branches. Data have been collected from the following references: CEMP dwarfs - Lucatello et al. (2003), Sivarani et al. (2004); CEMP subgiants - Aoki et al. (2002b), Cohen et al. (2003), Tsangarides (2005), Cohen et al. (2006), Thompson et al. (2008); CEMP giants - Aoki et al. (2001, 2002b), Barbuy et al. (2005), Masseron et al. (2010); CH subgiants - Luck \& Bond (1991), Pereira \& Junqueira (2003), Pereira \& Drake (2011); CH giants - Vanture (1992b,c), Kipper et al. (1996), Začs et al. (1998), Van Eck et al. (2001), Johnson \& Bolte (2004), Goswami et al. (2006), Pereira \& Drake (2009), Goswami \& Aoki (2010), Masseron et al. (2010); Ba dwarfs Allen \& Barbuy (2006); Ba giants - Allen \& Barbuy (2006), Pereira \& Drake (2009).

five mdBa stars have been classified based on chemical analyses (Luck \& Bond 1991; Junqueira \& Pereira 2001). Some works have presented different views of these five "mdBa" stars. $\mathrm{BD}+04^{\circ} 2466$ was confirmed as a $\mathrm{CH}$ giant by Pereira \& Drake (2009). Jorissen et al. (2005) considered HD 104340 and $\mathrm{BD}+03^{\circ} 2688$ as intrinsic Ba stars, instead of classical Ba stars belonging to a binary system, because they lie on the thermally pulsing AGB sequence and did not show any indication of orbital motion from radial velocity measurements. Jorissen et al. (2005) also doubt the binary nature of HD 55496 and HD 206983 since they had no, or inconclusive, radial velocity data.

For a wide range of $[\mathrm{Fe} / \mathrm{H}](-3<[\mathrm{Fe} / \mathrm{H}]<0),[l s / \mathrm{Fe}]$ has no dependence on $[\mathrm{Fe} / \mathrm{H}]$ for all classes of chemically peculiar binary stars, whereas $[h s / \mathrm{Fe}]$ shows a decreasing trend with increasing $[\mathrm{Fe} / \mathrm{H}]$ albeit with some scatter. The data of $\mathrm{G} 24-25$ are consisted with this trend indicating similar origins for these stars. This decreasing trend with $[\mathrm{Fe} / \mathrm{H}]$ becomes even more obvious in the $[h s / l s]$ versus (vs.) $[\mathrm{Fe} / \mathrm{H}]$ panel. In addition, there is a hint that the $[h s / l s]([h s / l s]=[h s / \mathrm{Fe}]-[l s / \mathrm{Fe}])$ ratios of

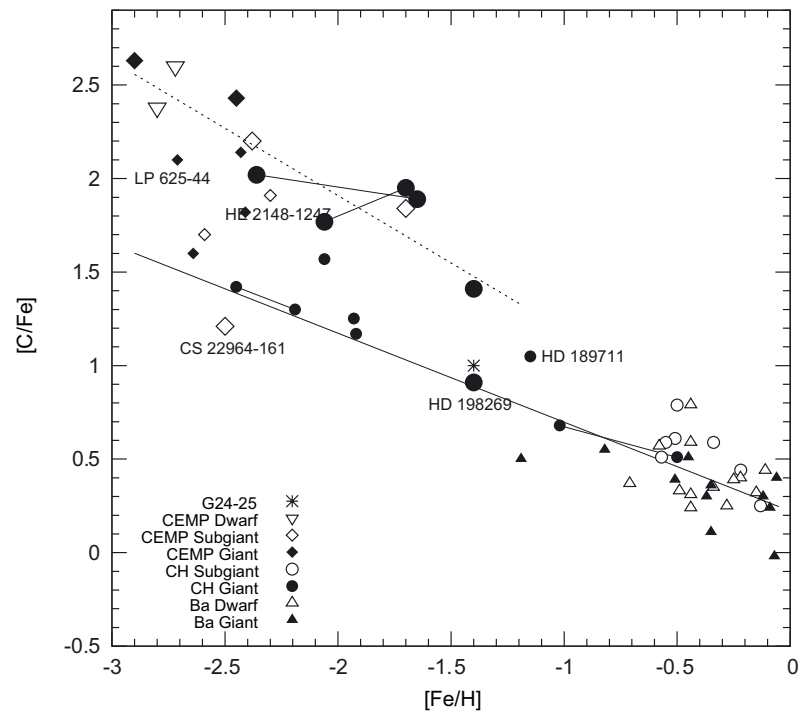

Fig. 9. Comparison of $[\mathrm{C} / \mathrm{Fe}]$ vs. $[\mathrm{Fe} / \mathrm{H}]$ for chemically peculiar binary stars. The big and small sizes of one particular symbol represent stars with $[\mathrm{Pb} / \mathrm{Ba}]>1$ and $[\mathrm{Pb} / \mathrm{Ba}]<1$, respectively.

giants are systematically higher than those of subgiants/dwarfs at $[\mathrm{Fe} / \mathrm{H}]>-0.5$, but further checks of the differences in the abundance analyses between giants and subgiants/dwarfs are needed.

According to Luck \& Bond (1991) and Busso et al. (2001), $[h s / l s]$ is an indicator of the neutron-exposure in the $s$-process nucleosynthesis: large $[h s / l s]$ ratios correspond to high neutronexposures. Therefore, the CEMP, $\mathrm{CH}$, and $\mathrm{Ba}$ stars can be described by the same scenario in different metallicity ranges where the neutron-exposure in these stars increases as the metallicity decreases. In agreement with this trend, Fig. 9 shows the $[\mathrm{C} / \mathrm{Fe}]$ ratio as a function of metallicity for the same objects as in Fig. 8. All chemically peculiar binary stars have a carbon enhancement that increases with decreasing $[\mathrm{Fe} / \mathrm{H}]$. The scatter in the $[\mathrm{C} / \mathrm{Fe}]$ ratios becomes significant for CEMP stars with $[\mathrm{Fe} / \mathrm{H}]<-2.0$. There is a tendency for the carbon enhancement to diverge into two branches as shown by the solid (low $[\mathrm{C} / \mathrm{Fe}]$ ) and dashed (high $[\mathrm{C} / \mathrm{Fe}]$ ) lines in Fig. 9. Among the high branch, 8 of 11 stars have $[\mathrm{Pb} / \mathrm{Ba}]>1.0$, but three stars (LP 625-44, HE 2148-1247, and HD 189711) have $[\mathrm{Pb} / \mathrm{Ba}]<1.0$; notably, HD 189711 has large uncertainties of $0.5 \mathrm{dex}$ in $[\mathrm{Pb} / \mathrm{Fe}]$ (Van Eck et al. 2003), and HE 2148-1247 is a typical CEMP-r/s star (Cohen et al. 2003). On the lower branch, most stars have $[\mathrm{Pb} / \mathrm{Ba}]<1.0$, except for two stars (CS 22964-161 and HD 198269) for which $[\mathrm{Pb} / \mathrm{Ba}]>1.0$. According to Thompson et al. (2008), CS 22964-161 is a triple system with a double-lined spectroscopic binary and a third component that might be responsible for its anomalous abundance pattern. The diverging branches of $[\mathrm{C} / \mathrm{Fe}]$ versus $[\mathrm{Fe} / \mathrm{H}]$ are also quite prominent in Fig. 8 of Pereira \& Drake (2009), who make no comment on this. Our star G 24-25 belongs to the lower branch. Careful inspection of the top panel of Fig. 8 shows evidence of the diverging branches in the $[h s / \mathrm{Fe}]$ vs. $[\mathrm{Fe} / \mathrm{H}]$ diagram: stars located above the dashed line correspond to the high branch in Fig. 9 (except for HD 209621, a CEMP- $r / s$ star), while those below the dashed line correspond to the low branch. Such diverging branches could also occur in the $[l s / \mathrm{Fe}]$ vs. $[\mathrm{Fe} / \mathrm{H}]$ diagram, but do not appear to do so, as seen in the middle panel, owing to the smaller effect of the neutronexposure and large scatter in the data. This similarity seems to be reasonable in the sense that the enhancements of $\mathrm{C}, \mathrm{Pb}, h s$, and 
probably $l s$ all correspond to the strength of neutron radiation flux but to a decreasing degree.

Masseron et al. (2010) completed a holistic study of the CEMP stars. They compiled abundances from analyses of high resolution spectra of 111 CEMP stars and 21 Ba stars, including both binary and non-binary stars, and covering nearly all the chemically peculiar binary stars selected in this work. For four stars (HD 26, HD 187861, HD 196944, and HD 224959), Masseron et al. (2010) give updated abundance results. Comparing the left panel of Fig. 5 in Masseron et al. (2010) ([Ce/Fe] vs. $[\mathrm{Fe} / \mathrm{H}]$, where $\mathrm{Ce}$ is a representative $h s$-process element) with the top panel of Fig. 8 in this work, we can see that $[\mathrm{Ce} / \mathrm{Fe}]$ shows a significant scatter at all metallicities and no obvious decreasing trend with increasing $[\mathrm{Fe} / \mathrm{H}]$, in contrast to our data for $[h s / \mathrm{Fe}]$ in Fig. 8. There are two possible reasons for this difference: the first is a statistical effect where $[h s / \mathrm{Fe}]$ is based on the average abundance of five $s$-process elements, which thus has a smaller the scatter; the second is that Masseron et al. (2010) adopted more stars, i.e. all CEMP- $s$ stars (the CEMP-low- $s$ included) in their Fig. 5, but we consider only the $s$-process enhanced binary stars.

\section{Conclusions}

On the basis of high resolution and high $\mathrm{S} / \mathrm{N}$ spectra, the abundances of carbon and 12 neutron-capture elements, including $\mathrm{Pb}$, are obtained for G 24-25. The C and s-process element abundances from the first, second, and third peaks show significant enhancements in this peculiar star with respect to the Sun and also with respect to a reference star G 16-20, which has similar atmospheric parameters as G 24-25. The abundance ratios $[\mathrm{Pb} / \mathrm{Fe}]=1.68$ and $[\mathrm{Pb} / \mathrm{Ba}]=0.33$ show that it is not a typical lead star (Van Eck et al. 2001), which are often found at low metallicity $([\mathrm{Fe} / \mathrm{H}]<-2)$ to have $[\mathrm{Pb} / \mathrm{Ba}]>1.0$. Owing to its binary signature, high radial velocity, and abundance pattern, we suggest that G 24-25 is a newly discovered $\mathrm{CH}$ subgiant with a clear detection of the $\mathrm{Pb}$ line at $\lambda 4057.8$.

Two simple AGB wind-accretion models have been adopted to predict the theoretical abundances of the $s$-process elements. The comparison of the observed abundances with the model predictions indicates that mass transfer via wind accretion across a binary system from its principal component, a low-mass AGB star, succeeds in reproducing the enhancements of the neutron-capture elements. When compared with other chemically peculiar binary stars in the literature, we have found that G 24-25 follows the general trend of $[s / \mathrm{Fe}],[h s / l s]$, and $[\mathrm{C} / \mathrm{Fe}]$ versus $[\mathrm{Fe} / \mathrm{H}]$ established for $\mathrm{Ba}$ stars, $\mathrm{CH}$ stars, and CEMP-s stars implying that there is a similar origin for these objects.

Acknowledgements. It is a pleasure to thank J. R. Shi and K. F. Tan for valuable discussions on the determination of lead abundances. We thank B. Zhang and X. J. Shen for the calculation of and discussions on AGB model predictions. We thank W. Aoki for providing the molecular line data for the blue $\mathrm{CN}$ band, and an anonymous referee for helpful comments and suggestions. This study is supported by the National Natural Science Foundation of China under grants No. 11073026 and 10821061, the key project of Chinese Academy of Sciences No. KJCX2-YW-T22, the National Basic Research Program of China (973 program) No. 2007CB815103/815403.

\section{References}

Allen, D. M., \& Barbuy, B. 2006, A\&A, 454, 895

Anstee, S. D., \& O'Mara, B. J. 1995, MNRAS, 276, 859

Aoki, W., Ryan, S. G., Norris, J. E., et al. 2001, ApJ, 561, 346

Aoki, W., Norris, J. E., Ryan, S. G., Beers, T. C., \& Ando, H. 2002a, PASJ, 54, 933
Aoki, W., Ryan, S. G., Norris, J. E., et al. 2002b, ApJ, 580, 1149 Aoki, W., Ryan, S. G., Iwamoto, N., et al. 2003, ApJ, 592, L67 Asplund, M., Grevesse, N., Sauval, A. J., \& Scott, P. 2009, ARA\&A, 47, 481 Bao, Z. Y., Beer, H., Käppeler, F., et al. 2000, Atomic Data and Nuclear Data Tables, 76, 70

Barbuy, B., Spite, M., Spite, F., et al. 2005, A\&A, 429, 1031

Barklem, P. S., \& O'Mara, B. J. 1997, MNRAS, 290, 102

Barklem, P. S., \& O'Mara, B. J. 2000, MNRAS, 311, 535

Barklem, P. S., Piskunov, N., \& O'Mara, B. J. 2000, A\&AS, 142, 467

Barklem, P. S., Christlieb, N., Beers, T. C., et al. 2005, A\&A, 439, 129

Beers, T. C., \& Christlieb, N. 2005, ARA\&A, 43, 531

Biémont, E., Grevesse, N., Hannaford, P., \& Lowe, R. M. 1981, ApJ, 248, 867

Biémont, E., Grevesse, N., Hannaford, P., \& Lowe, R. M. 1989, A\&A, 222, 307

Biémont, E., Garnir, H. P., Palmeri, P., Li, Z. S., \& Svanberg, S. 2000, MNRAS, 312,116

Bisterzo, S., Gallino, R., Straniero, O., et al. 2006, Mem. S. A. It., 77, 985

Bisterzo, S., Gallino, R., Straniero, O., Cristallo, S., \& Käppeler, F. 2010, MNRAS, 404, 1529

Bisterzo, S., Gallino, R., Straniero, O., Cristallo, S., \& Käppeler, F. 2011, MNRAS, 418, 284

Busso, M., Gallino, R., Lambert, D. L., Travaglio, C., \& Smith, V. V. 2001, ApJ, 557,802

Cayrel, R. 1988, The Impact of Very High S/N Spectroscopy on Stellar Physics, 132,345

Chieffi, A., Limongi, M., \& Straniero, O. 1998, ApJ, 502, 737

Cohen, J. G., Christlieb, N., Qian, Y.-Z., \& Wasserburg, G. J. 2003, ApJ, 588, 1082

Cohen, J. G., McWilliam, A., Shectman, S., et al. 2006, AJ, 132, 137

Cristallo, S., Straniero, O., Gallino, R., et al. 2009, ApJ, 696, 797

Cristallo, S., Piersanti, L., Straniero, O., et al. 2011, ApJS, 197, 17

Corliss, C. H., \& Bozman, W. R. 1962, Experimental Transition Probabilities for Spectral lines of Seventy elements, NBS Monograph 53

Cowan, J. J., Sneden, C., Burles, S., et al. 2002, ApJ, 572, 861

Cowley, C. R., \& Corliss, C. H. 1983, MNRAS, 203, 651

Cui, W.-Y., Zhang, J., Zhu, Z.-Z., \& Zhang, B. 2010, ApJ, 708, 51

Davidson, M. D., Snoek, L. C., Volten, H., \& Doenszelmann, A. 1992, A\&A, 255,457

Den Hartog, E. A., Lawler, J. E., Sneden, C., \& Cowan, J. J. 2003, ApJS, 148, 543

Den Hartog, E. A., Lawler, J. E., Sneden, C., \& Cowan, J. J. 2006, ApJS, 167, 292

Gallino, R., Arlandini, C., Busso, M., et al. 1998, ApJ, 497, 388

Goswami, A., \& Aoki, W. 2010, MNRAS, 404, 253

Goswami, A., Aoki, W., Beers, T. C., et al. 2006, MNRAS, 372, 343

Gratton, R. G., \& Sneden, C. 1994, A\&A, 287, 927

Hannaford, P., Lowe, R. M., Grevesse, N., Biemont, E., \& Whaling, W. 1982, ApJ, 261, 736

Hibbert, A., Biémont, E., Godefroid, M., \& Vaeck, N. 1993, A\&AS, 99, 179

Johnson, J. A., \& Bolte, M. 2004, ApJ, 605, 462

Jorissen, A., Začs, L., Udry, S., Lindgren, H., \& Musaev, F. A. 2005, A\&A, 441, 1135

Junqueira, S., \& Pereira, C. B. 2001, AJ, 122, 360

Karakas, A., \& Lattanzio, J. C. 2007, PASA, 24, 103

Kipper, T., Jorgensen, U. G., Klochkova, V. G., \& Panchuk, V. E. 1996, A\&A, 306, 489

Kurucz, R. L. 1993, SYNTHE Spectrum Synthesis Programs and Line Data, Kurucz CD-ROM, No. 18 (Cambridge, Mass.: Smithsonian Astrophysical Observatory)

Latham, D. W., Stefanik, R. P., Torres, G., et al. 2002, AJ, 124, 1144

Lawler, J. E., Bonvallet, G., \& Sneden, C. 2001, ApJ, 556, 452

Lawler, J. E., Sneden, C., Cowan, J. J., Ivans, I. I., \& Den Hartog, E. A. 2009, ApJS, 182, 51

Ljung, G., Nilsson, H., Asplund, M., \& Johansson, S. 2006, A\&A, 456, 1181

Lu, P. K., Demarque, P., van Altena, W., McAlister, H., \& Hartkopf, W. 1987, AJ, 94, 1318

Lucatello, S., Gratton, R., Cohen, J. G., et al. 2003, AJ, 125, 875

Lucatello, S., Tsangarides, S., Beers, T. C., et al. 2005, ApJ, 625, 825

Luck, R. E., \& Bond, H. E. 1982, ApJ, 259, 792

Luck, R. E., \& Bond, H. E. 1991, ApJS, 77, 515

Mashonkina, L., \& Gehren, T. 2000, A\&A, 364, 249

Mashonkina, L., Ryabchikova, T., Ryabtsev, A., \& Kildiyarova, R. 2009, A\&A, 495, 297

Mashonkina, L., Christlieb, N., Barklem, P. S., et al. 2010, A\&A, 516, A46

Masseron, T., Johnson, J. A., Plez, B., et al. 2010, A\&A, 509, A93

McClure, R. D., \& Woodsworth, A. W. 1990, ApJ, 352, 709

Meggers, W. F., Corliss, C. H., \& Scribner, B. F. 1975, Tables of spectral-line intensities. Parts I and II, NBS Monogr., 145

Mennessier, M. O., Luri, X., Figueras, F., et al. 1997, A\&A, 326, 722 
S. Liu et al.: Abundances of neutron-capture elements in G 24-25

Nissen, P. E., \& Schuster, W. J. 2010, A\&A, 511, L10

Nissen, P. E., \& Schuster, W. J. 2011, A\&A, 530, A15

Nissen, P. E. Lambert, D. L., Primas, F. \& Smith, V. V. 1999, A\&A, 348, 211

Palmeri, P., Quinet, P., Wyart, J.-F., \& Biémont, E. 2000, Phys. Scr., 61, 323

Pereira, C. B., \& Drake, N. A. 2009, A\&A, 496, 791

Pereira, C. B., \& Drake, N. A. 2011, AJ, 141, 79

Pereira, C. B., \& Junqueira, S. 2003, A\&A, 402, 1061

Pourbaix, D., Tokovinin, A. A., Batten, A. H., et al. 2004, A\&A, 424, 727

Preston, G. W., \& Sneden, C. 2001, AJ, 122, 1545

Reddy, B. E., Parthasarathy, M., Gonzalez, G., \& Bakker, E. J. 1997, A\&A, 328, 331

Sivarani, T., Bonifacio, P., Molaro, P., et al. 2004, A\&A, 413, 1073

Smith, V. V., Coleman, H., \& Lambert, D. L. 1993, ApJ, 417, 287

Sneden, C., \& Parthasarathy, M. 1983, ApJ, 267, 757
Sneden, C., McWilliam, A., Preston, G. W., et al. 1996, ApJ, 467, 819 Straniero, O., Gallino, R., Busso, M., et al. 1995, ApJ, 440, L85

Thompson, I. B., Ivans, I. I., Bisterzo, S., et al. 2008, ApJ, 677, 556 Tsangarides, S. A. 2005, Ph.D. Thesis

Tsangarides, S., Ryan, S. G., \& Beers, T. C. 2004, Mem. S. A. It., 75, 772

Unsöld, A. 1955, Physik der Sternatmosphären (Berlin: Springer Verlag)

Van Eck, S., Goriely, S., Jorissen, A., \& Plez, B. 2001, Nature, 412, 793

Van Eck, S., Goriely, S., Jorissen, A., \& Plez, B. 2003, A\&A, 404, 291

Vanture, A. D. 1992a, AJ, 103, 2035

Vanture, A. D. 1992b, AJ, 104, 1986

Vanture, A. D. 1992c, AJ, 104, 1997

Začs, L., Nissen, P. E., \& Schuster, W. J. 1998, A\&A, 337, 216

Zhang, B., Ma, K., \& Zhou, G. 2006, ApJ, 642, 1075 
Table 2. Atomic data and equivalent widths of spectral lines, as well as the derived abundances for G 24-25 and G 16-20.

\begin{tabular}{|c|c|c|c|c|c|c|c|c|}
\hline \multirow{2}{*}{$\begin{array}{l}\text { Wavelength } \\
\text { (§) }\end{array}$} & \multirow[t]{2}{*}{ Elem. } & \multirow{2}{*}{$\begin{array}{l}\text { E. P. } \\
(\mathrm{eV})\end{array}$} & \multirow[t]{2}{*}{$\log g f$} & \multirow[t]{2}{*}{ Ref. } & \multicolumn{2}{|c|}{ G $24-25$} & \multicolumn{2}{|c|}{ G 16-20 } \\
\hline & & & & & $E W(\mathrm{~m} \AA)$ & $\log \varepsilon$ & $E W(\mathrm{~m} \AA)$ & $\log \varepsilon$ \\
\hline 4932.049 & $\mathrm{C}_{\mathrm{I}}$ & 7.68 & -1.68 & 1 & 14.6 & 8.08 & & \\
\hline 5052.167 & $\mathrm{C}_{\mathrm{I}}$ & 7.68 & -1.30 & 1 & 23.8 & 7.97 & 1.2: & 6.58: \\
\hline 5380.337 & $\mathrm{C}_{\mathrm{I}}$ & 7.68 & -1.62 & 1 & 14.1 & 8.02 & $\ldots$ & $\ldots$ \\
\hline 4607.340 & Sr I & 0.00 & 0.28 & 2 & 18.0 & 1.96 & 5.7 & 1.19 \\
\hline 4077.714 & $\mathrm{Sr}_{\text {II }}$ & 0.00 & 0.17 & 2 & 234.8 & 1.79 & 154.3 & 1.09 \\
\hline 4398.010 & $\mathrm{Y}_{\text {II }}$ & 0.13 & -1.00 & 3 & 50.9 & 1.58 & 18.3 & 0.56 \\
\hline 4883.690 & $\mathrm{Y}_{\mathrm{II}}$ & 1.08 & 0.07 & 3 & 56.1 & 1.52 & 25.0 & 0.60 \\
\hline 4900.110 & $\mathrm{Y}_{\text {II }}$ & 1.03 & -0.09 & 3 & 56.2 & 1.63 & 23.5 & 0.67 \\
\hline 5087.430 & $\mathrm{Y}_{\text {II }}$ & 1.08 & -0.17 & 3 & 43.4 & 1.45 & 15.0 & 0.54 \\
\hline 5123.220 & $\mathrm{Y}_{\mathrm{II}}$ & 0.99 & -0.83 & 3 & 22.0 & 1.51 & 4.5 & 0.53 \\
\hline 5200.420 & $\mathrm{Y}_{\mathrm{II}}$ & 0.99 & -0.57 & 3 & 30.8 & 1.46 & 8.8 & 0.58 \\
\hline 5205.730 & $\mathrm{Y}_{\text {II }}$ & 1.03 & -0.34 & 3 & 39.3 & 1.47 & 14.7 & 0.64 \\
\hline 5402.780 & $\mathrm{Y}_{\text {II }}$ & 1.84 & -0.44 & 3 & 8.6 & 1.44 & $\ldots$ & $\ldots$ \\
\hline 4687.800 & $\mathrm{Zr}$ & 0.73 & 0.55 & 4 & 4.7 & 2.14 & $\ldots$ & $\ldots$ \\
\hline 4050.330 & $\mathrm{Zr}_{\text {II }}$ & 0.71 & -1.06 & 5 & 23.2 & 2.06 & 11.5 & 1.47 \\
\hline 4090.510 & $\mathrm{Zr}_{\text {II }}$ & 0.76 & -1.01 & 6 & 26.7 & 2.14 & $\ldots$ & $\ldots$ \\
\hline 4161.200 & $\mathrm{Zr}_{\text {II }}$ & 0.71 & -0.59 & 5 & 46.7 & 2.15 & 24.1 & 1.39 \\
\hline 4208.990 & Zr II & 0.71 & -0.51 & 5 & 47.0 & 2.07 & 26.1 & 1.36 \\
\hline 4258.050 & Zr II & 0.56 & -1.20 & 5 & 27.2 & 2.13 & $\ldots$ & $\ldots$ \\
\hline 5350.080 & $\mathrm{Zr}$ II & 1.83 & -1.24 & 6 & 2.9 & 2.20 & $\ldots$ & $\ldots$ \\
\hline 5350.310 & $\mathrm{Zr}$ II & 1.77 & -1.16 & 5 & 4.2 & 2.22 & $\ldots$ & $\ldots$ \\
\hline 5853.688 & Ba II & 0.60 & -0.91 & 7 & 94.6 & 2.15 & 29.5 & 0.55 \\
\hline 6141.727 & Ba II & 0.70 & -0.03 & 7 & 163.1 & 2.08 & 69.0 & 0.53 \\
\hline 6496.908 & Ba II & 0.60 & -0.41 & 7 & 142.5 & 2.13 & 61.6 & 0.64 \\
\hline 4526.110 & La II & 0.77 & -0.59 & 8 & 15.0 & 1.22 & $\ldots$ & $\ldots$ \\
\hline 4662.510 & La II & 0.00 & -1.24 & 8 & 22.8 & 1.28 & $\ldots$ & $\ldots$ \\
\hline 4748.730 & La II & 0.93 & -0.54 & 8 & 14.7 & 1.23 & $\ldots$ & $\ldots$ \\
\hline 4920.960 & La II & 0.13 & -0.58 & 8 & 48.8 & 1.38 & 10.1 & 0.06 \\
\hline 4921.780 & La II & 0.24 & -0.45 & 8 & 45.2 & 1.27 & 10.6 & 0.07 \\
\hline 4053.490 & $\mathrm{Ce}_{\text {II }}$ & 0.00 & -0.61 & 9 & 29.5 & 1.71 & $\ldots$ & $\ldots$ \\
\hline 4062.230 & $\mathrm{Ce}_{\text {II }}$ & 1.37 & 0.30 & 10 & 19.6 & 1.88 & 1.2: & $0.36:$ \\
\hline 4083.220 & $\mathrm{Ce}_{\text {II }}$ & 0.70 & 0.27 & 9 & 36.1 & 1.70 & $\ldots$ & $\ldots$ \\
\hline 4117.290 & $\mathrm{Ce}_{\text {II }}$ & 0.74 & -0.45 & 9 & 11.3 & 1.69 & $\ldots$ & $\ldots$ \\
\hline 4118.140 & $\mathrm{Ce}_{\text {II }}$ & 0.70 & 0.13 & 9 & 32.4 & 1.74 & $\ldots$ & $\ldots$ \\
\hline 4120.830 & $\mathrm{Ce}_{\text {II }}$ & 0.32 & -0.37 & 9 & 31.9 & 1.85 & $\ldots$ & $\ldots$ \\
\hline 4127.360 & $\mathrm{Ce}_{\text {II }}$ & 0.68 & 0.31 & 9 & 40.9 & 1.76 & $\ldots$ & $\ldots$ \\
\hline 4153.130 & $\mathrm{Ce}_{\text {II }}$ & 0.23 & -0.80 & 9 & 16.7 & 1.74 & $\ldots$ & $\ldots$ \\
\hline 4222.600 & $\mathrm{Ce}_{\text {II }}$ & 0.12 & -0.15 & 9 & 46.6 & 1.80 & 6.6 & 0.28 \\
\hline 4427.920 & $\mathrm{Ce}_{\text {II }}$ & 0.54 & -0.41 & 9 & 19.2 & 1.71 & . & $\ldots$ \\
\hline 4460.230 & $\mathrm{Ce}_{\text {II }}$ & 0.47 & 0.28 & 10 & $\ldots$ & $\ldots$ & 10.7 & 0.41 \\
\hline 4483.900 & $\mathrm{Ce}_{\text {II }}$ & 0.86 & 0.10 & 9 & 29.0 & 1.79 & $\ldots$ & $\ldots$ \\
\hline 4523.080 & $\mathrm{Ce}_{\text {II }}$ & 0.52 & -0.08 & 9 & $\ldots$ & $\ldots$ & 3.1 & 0.24 \\
\hline 4539.740 & $\mathrm{Ce}_{\text {II }}$ & 0.33 & -0.08 & 9 & 42.5 & 1.78 & 5.4 & 0.30 \\
\hline 4560.960 & $\mathrm{Ce}_{\text {II }}$ & 0.68 & -0.26 & 9 & 23.5 & 1.82 & $\ldots$ & $\ldots$ \\
\hline 4562.360 & $\mathrm{Ce}_{\text {II }}$ & 0.48 & 0.21 & 9 & 50.6 & 1.85 & 7.0 & 0.29 \\
\hline 4565.840 & $\mathrm{Ce}_{\text {II }}$ & 1.09 & 0.07 & 9 & 18.2 & 1.74 & $\ldots$ & $\ldots$ \\
\hline 4628.160 & $\mathrm{Ce}_{\text {II }}$ & 0.52 & 0.14 & 9 & 46.1 & 1.83 & $\ldots$ & $\ldots$ \\
\hline 5044.030 & $\mathrm{Ce}_{\text {II }}$ & 1.21 & -0.14 & 9 & 10.4 & 1.72 & $\ldots$ & $\ldots$ \\
\hline 5187.450 & $\mathrm{Ce}_{\text {II }}$ & 1.21 & 0.17 & 9 & 18.1 & 1.71 & $\ldots$ & $\ldots$ \\
\hline 5274.230 & $\mathrm{Ce}_{\text {II }}$ & 1.04 & 0.13 & 9 & 23.3 & 1.72 & $\ldots$ & $\ldots$ \\
\hline 5330.540 & $\mathrm{Ce}_{\text {II }}$ & 0.87 & -0.40 & 9 & 12.4 & 1.72 & $\ldots$ & $\ldots$ \\
\hline 5468.380 & $\mathrm{Ce}_{\text {II }}$ & 1.40 & -0.07 & 9 & 8.9 & 1.75 & $\ldots$ & $\ldots$ \\
\hline 5610.246 & $\mathrm{Ce}_{\text {II }}$ & 1.05 & -0.74 & 10 & 6.0 & 1.85 & $\ldots$ & $\ldots$ \\
\hline 5322.800 & $\operatorname{Pr}_{I I}$ & 0.48 & -0.32 & 11 & syn & 1.28 & syn & $<-0.38$ \\
\hline 4012.700 & $\mathrm{Nd}$ II & 0.00 & -0.74 & 12 & 27.0 & 1.56 & 4.3 & 0.35 \\
\hline 4051.150 & $\mathrm{Nd}_{\text {II }}$ & 0.38 & -0.30 & 13 & 28.5 & 1.54 & 6.4 & 0.48 \\
\hline 4232.380 & $\mathrm{Nd}_{\text {II }}$ & 0.06 & -0.47 & 13 & 35.1 & 1.53 & $\ldots$ & $\ldots$ \\
\hline 4542.603 & $\mathrm{Nd}_{\text {II }}$ & 0.74 & -0.28 & 13 & 17.2 & 1.49 & 2.4 & 0.34 \\
\hline 4645.770 & Nd II & 0.56 & -0.76 & 13 & 10.0 & 1.49 & $\ldots$ & $\ldots$ \\
\hline 5089.830 & $\mathrm{Nd}_{\text {II }}$ & 0.28 & -1.40 & 2 & 5.5 & 1.53 & $\ldots$ & $\ldots$ \\
\hline 5092.780 & $\mathrm{Nd}_{\text {II }}$ & 0.30 & -0.61 & 13 & 21.4 & 1.46 & 2.6 & 0.29 \\
\hline 5192.620 & $\mathrm{Nd}_{\text {II }}$ & 1.14 & 0.27 & 13 & 25.6 & 1.53 & 3.6 & 0.33 \\
\hline 5234.210 & $\mathrm{Nd}_{\text {II }}$ & 0.55 & -0.51 & 13 & 21.2 & 1.59 & $\ldots$ & $\ldots$ \\
\hline
\end{tabular}


Table 2. continued.

\begin{tabular}{|c|c|c|c|c|c|c|c|c|}
\hline \multirow{2}{*}{$\begin{array}{l}\text { Wavelength } \\
(\AA)\end{array}$} & \multirow[t]{2}{*}{ Elem. } & \multirow{2}{*}{$\begin{array}{l}\text { E. P. } \\
(\mathrm{eV})\end{array}$} & \multirow[t]{2}{*}{$\log g f$} & \multirow[t]{2}{*}{ Ref. } & \multicolumn{2}{|c|}{$\mathrm{G} 24-25$} & \multicolumn{2}{|c|}{ G $16-20$} \\
\hline & & & & & $E W(\mathrm{~m} \AA)$ & $\log \varepsilon$ & $E W(\mathrm{~m} \AA)$ & $\log \varepsilon$ \\
\hline 5249.600 & $\mathrm{Nd}$ II & 0.98 & 0.20 & 13 & 26.9 & 1.47 & $\ldots$ & $\ldots$ \\
\hline 5255.510 & $\mathrm{Nd}_{\text {II }}$ & 0.20 & -0.67 & 13 & 25.8 & 1.53 & $\ldots$ & $\ldots$ \\
\hline 5311.480 & $\mathrm{Nd}_{\text {II }}$ & 0.99 & -0.42 & 13 & 8.3 & 1.44 & $\ldots$ & $\ldots$ \\
\hline 5319.820 & $\mathrm{Nd}_{\text {II }}$ & 0.55 & -0.21 & 12 & 31.1 & 1.55 & 3.6 & 0.20 \\
\hline 5385.890 & $\mathrm{Nd}_{\text {II }}$ & 0.74 & -0.82 & 12 & 7.6 & 1.54 & $\ldots$ & $\ldots$ \\
\hline 4244.700 & Sm II & 0.28 & -0.73 & 14 & 10.1 & 0.93 & $\ldots$ & $\ldots$ \\
\hline 4434.320 & Sm II & 0.38 & -0.26 & 15 & 24.6 & 1.06 & 4.6 & -0.04 \\
\hline 4458.520 & Sm II & 0.10 & -0.78 & 15 & 14.3 & 0.97 & $\ldots$ & $\ldots$ \\
\hline 4519.630 & Sm II & 0.54 & -0.43 & 14 & 14.8 & 1.07 & $\ldots$ & $\ldots$ \\
\hline 4523.910 & Sm II & 0.43 & -0.58 & 14 & 13.7 & 1.06 & $\ldots$ & $\ldots$ \\
\hline 4566.210 & Sm II & 0.33 & -0.92 & 15 & 9.6 & 1.11 & $\ldots$ & $\ldots$ \\
\hline 4642.230 & Sm II & 0.38 & -0.52 & 14 & 12.1 & 0.93 & 2.4 & -0.09 \\
\hline 4674.600 & Sm II & 0.18 & -0.56 & 15 & 15.6 & 0.90 & 3.7 & -0.07 \\
\hline 4687.180 & $\mathrm{Sm}_{\text {II }}$ & 0.04 & -1.17 & 15 & 9.7 & 1.07 & $\ldots$ & $\ldots$ \\
\hline 4129.700 & Eu II & 0.00 & 0.22 & 16 & syn & -0.25 & syn & -0.50 \\
\hline 6645.130 & Eu II & 1.38 & 0.20 & 16 & syn & $-0.30:$ & syn & -0.56 : \\
\hline 4191.080 & Gd II & 0.43 & -0.48 & 17 & syn & 0.84 & syn & 0.08: \\
\hline 4057.810 & $\mathrm{~Pb}_{\mathrm{I}}$ & 1.32 & -0.22 & 18 & syn & 2.28 & syn & $<0.60$ \\
\hline
\end{tabular}

Notes. This table contains the following information: wavelength in angstroms (Col. 1), element identification (Col. 2), excitation potential (Col. 3), $\log$ of the oscillator strength (Col. 4), reference for $\log g f(\mathrm{Col} .5)$, measured equivalent width in milli-angstroms and derived abundance of $\mathrm{G} 24-25$ (Cols. 6 and 7), measured equivalent width in milli-angstroms and derived abundance of G 16-20 (Cols. 8 and 9).

References. (1) Hibbert et al. (1993); (2) Gratton \& Sneden (1994); (3) Hannaford et al. (1982); (4) Biémont et al. (1981); (5) Ljung et al. (2006); (6) Cowley \& Corliss (1983); (7) Davidson et al. (1992); (8) Lawler et al. (2001); (9) Lawler et al. (2009); (10) Palmeri et al. (2000); (11) Mashonkina et al. (2009); (12) Meggers et al. (1975); (13) Den Hartog et al. (2003); (14) Biémont et al. (1989); (15) Corliss \& Bozman (1962); (16) Mashonkina \& Gehren (2000); (17) Den Hartog et al. (2006); (18) Biémont et al. (2000). 